Using the RP, 3.8\% of all patients would be 'inappropriately' redirected but if decision to re-direct were based only on TN assessment this reduced to $3.6 \%$ with a $58 \%$ reduction in $\mathrm{T} 4$ and 5 patients being seen 'unnecessarily' in the PED.

Conclusions Over $30 \%$ of T4 and 5 patients presenting to the ED would be appropriate for re-direction to primary care services $-12 \%$ of all attendances. This would be considerably higher if a more inclusive RP was created to account for trivial and non-urgent presentations to ED. TN assessment safely and accurately identifies patients requiring $\mathrm{PED}$ specific care.

\section{G101(P) TIME FOR BETTER NORMAL RANGES? THE IMPACT OF DISCONTINUITIES IN NORMAL RANGES FOR HEART AND RESPIRATORY RATE IN PAEDIATRIC A\&E}

${ }^{1} \mathrm{AJ}$ McArdle, ${ }^{1} \mathrm{PA}$ Stilwell, ${ }^{2} \mathrm{H}$ Kwong, ${ }^{3} \mathrm{M}$ Blair. ${ }^{1}$ Department of Paediatrics, Northwick Park Hospital, London, UK; ${ }^{2}$ Faculty of Medicine, Imperial College, London, UK; ${ }^{3}$ Department of Paediatrics, Imperial College, London, UK

\subsection{6/archdischild-2015-308599.100}

Aims Clinical assessment in A\&E is heavily influenced by physiological parameters. However, paediatric normal ranges have large discontinuities and are based on poor evidence. Evidencebased centile charts (Fleming et al, 2010) demonstrate striking disagreements with widely used ranges.

We studied the change in proportion of children with high triage observations by age, and investigated whether discontinuities at age transitions are associated with discrete changes in management.

Methods We obtained details of 14,831 children attending our paediatric A\&E in 2013 (excluding psychosocial and trauma) and extracted missing triage observations from scanned records. We determined whether CRP was measured for each patient.

Though our department uses PEWS normal ranges (transitions at age 1, 5 and 12) we used the more widespread APLS ranges for classification. High triage observations were determined by APLS, centile charts (90th centile) and derived normal ranges. For APLS, steps in proportions were determined with discontinuous linear regression.

Separately, we used spline regression models to test for the presence of steps at age 1,5 and 12 in length of stay, probability of admission and CRP measurement, with subgroup analysis of children $<8$ y with triage category "fever".

For children presenting with wheeze, we compared the proportions receiving burst therapy or intravenous treatment prior to and after the first, fifth and twelfth birthdays.

Results The proportion of high triage observations by APLS showed significant steps at age 1,2, 5 and 12. 16\% of APLS classifications mismatched classification by centile chart. With derived age-specific normal ranges, this fell to $2 \%$.

We found no evidence of discrete changes in length of stay, probability of admission or measurement of CRP at the age boundaries. Similarly, in the febrile children and wheeze subsets we found no steps.

Conclusions The APLS normal ranges create large steps in the proportion of children with high observations at age transitions. However, we found no evidence of effects on management in this large dataset, nor in subgroups where observations contribute strongly to mangement.

Nonetheless, given the better performance of newer ranges, we encourage their further trial.

\section{G102(P) THE CHARACTERISTICS OF DRUG AND ALCOHOL- RELATED PRESENTATIONS TO THE EMERGENCY DEPARTMENT AMONGST ADOLESCENTS}

SS Patwardhan, F Norris, ED Edwards. Department of Paediatrics, Morriston Hospital, ABMU Health Board, Swansea, UK

\subsection{6/archdischild-2015-308599.101}

Introduction Concerns have been raised about the increasing presentation of children and young people (CYP) to the Emergency Departments (EDs) having taken recreational drugs or alcohol.

Aims The aims of this study are to estimate the proportion of drug and alcohol-related presentations to a large ED and to identify associated patient and presentation characteristics.

Methods A retrospective analysis of patients aged 12-18 years attending a large ED from January 2012-December 2012 was carried out. Characteristics of all presentations related to alcohol and recreational drug use were recorded.

Results 167 patients were included in the study. 35\% of patients were under 16 years of age, over $50 \%$ were female and over half of the presentations were at night or weekends. $51 \%$ presented with an altered level of consciousness, other presentations included respiratory or circulatory compromise, agitation, vomiting and injuries. Alcohol was the commonest substance taken seen in over three quarters of presentations, 38\% had taken illegal drugs and $18.5 \%$ admitted to taking both drug and alcohol. $78 \%$ presented to the ED via an ambulance. Investigations were performed in approximately half of the patients and included CT (0.5\%), ECG (23\%), blood tests (22\%) and urine toxicology $(4.3 \%)$. More than two-thirds did not receive any treatment but $16 \%$ of those attending the ED were admitted, mainly because of the effects of alcohol. 49\% of admitted patients were under 16 years of age.

Conclusion The number and presentations of alcohol and drug related attendances present a risk to CYP and is a burden to the ED and paediatric inpatient services. Effective intervention and preventative strategies are needed to reduce drug and alcohol related behaviour and co- morbidity.

\section{G103(P) ANALYSIS OF CONJUNCTIVITIS MANAGEMENT IN PAEDIATRIC EMERGENCY DEPARTMENT}

P McCrossan, S Deiratany, I Okafor, R McNamara. Emergency Department, Children's University Hospital, Temple Street, Dublin, Ireland

\subsection{6/archdischild-2015-308599.102}

Aims The aim of this study is to demonstrate an evidence based clinical guideline for the management of conjunctivitis and to illustrate the performance of a paediatric emergency department in its management of presentations of conjunctivitis.

Methods An evidenced based clinical guideline was developed. This guideline was then used as a set standard by which to assess the performance of the emergency department at CUH in its management of conjunctivitis. Data was collected retrospectively. All patients who attended CUH (children's university hospital) diagnosed with conjunctivitis, between 01/06/14-31/08/14 were used for analysis. The emergency department notes for these patients were examined and details of their presentation and subsequent management was noted. These details were collated and compared with the set standard outlined by the new clinical guideline. 
Results Mean age at presentation is 5.2 years (SD 1.8) [one month- 15 years]. During the sampled time frame (01/06/14-31/ $08 / 14$ ) there were a total of 54 presentations with a discharge diagnosis of conjunctivitis. $86 \%(25 / 29)$ were correctly diagnosed bacterial conjunctivitis. 52\% (13/25) were correctly diagnosed allergic conjunctivitis. 30 of the patients had documented features of bacterial conjunctivitis. 40\% (12/30) had an eye swabs taken. A total of 13 eye swabs were taken of which only one was indicated. $86 \%$ appropriately treated with topical antibiotics. $85 \%$ appropriately treated with antihistamines. $7 \%(3 / 41)$ were inappropriately treated with antihistamines. 28\% $(15 / 54)$ were referred for specialist opinion.

Discussion Conjunctivitis is a common presentation to a paediatrics emergency department. Indiscriminate use of microbiological investigations is a significant financial burden to the hospital and one, which can be easily eradicated. Prescribing of topical antibiotics or oral antihistamines should be based on sound clinical grounds so as to avoid unnecessary medicating of patients. Specialist referrals should be well considered.

\section{G104(P) RESUSCITATION EXPERIENCE OF PROFESSIONALS AT A DISTRICT GENERAL HOSPITAL (DGH)}

S Saeed, M Joishy. Paediatric Department, Ysbyty Gwynedd Hospital, Bangor, UK

\subsection{6/archdischild-2015-308599.103}

Introduction The caseload of critically ill patients at a DGH is usually smaller compared to tertiary hospitals. There are some concerns that modern centralisation of services may deskill clinicians at DGHs. Technology Enhanced Learning has an important role to play in improving training. We aimed to analyse professionals' experience, their role and confidence in performing resuscitation and also their opinion about simulation training.

Method We conducted a survey at Ysbyty Gwynedd to determine experience of medical and nursing colleagues in managing critically ill children and neonates over last 6 months. A questionnaire asking various aspects of resuscitation was sent out to nursing colleagues and paediatric doctors working at all levels. Of the 50 distributed questionnaires, 40 were completed and returned to us.

Results While only 30\% had exposure to resuscitation more than twice over preceding 6 months period, approximately $35 \%$ had once or no experience. Those who had exposure more than twice felt confident in performing resuscitation. While only $10 \%$ were involved in full cardiopulmonary resuscitation, about $30 \%$ were involved in airway and breathing and another 20\% in circulation. Over $90 \%$ stated that they would like to have regular simulation training at least monthly as they felt it will help in coping with actual resuscitation.

Conclusion There is limited data available in literature to determine frequency of managing critically ill children at individual level. This study shows that most health professionals have limited exposure to resuscitation situations at a DGH and nearly all paediatric staff wishes to receive regular simulation training.

\section{G105(P) YOUTH VIOLENCE: AN EDUCATIONAL SOLUTION?}

${ }^{1} \mathrm{CM}$ Reeder, ${ }^{2} \mathrm{CHN}$ Johnson. 'Medical School, University of Liverpool, Liverpool, UK; ${ }^{2}$ Medical School, University of Sheffield, Sheffield, UK

10.1136/archdischild-2015-308599.104
Background Violent/knife crime remains a significant public health challenge in the UK and Europe. Young people are disproportionately affected. Both physical injury in the short term and long term psychiatric conditions such as behavioural problems, post traumatic stress disorder, and substance misuse can result. ${ }^{1}$ Assault with a sharp instrument accounted for 3849 UK admissions to hospital last year $^{2}$ and so interventions which can reduce levels of violent crime and improve outcomes of victims of violent crimes are needed. StreetDoctors is a registered charity where basic life support and haemorrhage control sessions are taught to at risk young people in order to reduce mortality and morbidity from violent crimes. Since 2008 StreetDoctors has grown year on year to the point of expanding to 13 universities. Thanks to the hard work and dedication of the medical student volunteers we have taught well above 2500 young people ages 12-18.

Method A national approach was used to survey whether the young people were benefitting from our teaching. A pre and post teaching questionnaire was filled out by the young people to assess prior knowledge and knowledge gained

Results There was an Increase of $14.75 \%$ of young people knowing how to deal with someone who is passed out but breathing and an increase of $35.62 \%$ of young people knowing what to do when they found someone bleeding. A mean increase of 0.86 and of 0.92 points on a 1-10 scale answering the questions "how likely are you to help someone who's been stabbed/ is unconscious" respectively was also found.

Discussion This is the first time data has been collected across the country and our results show that the basic life support and haemorrhage control teaching we provide is having a positive impact on both the knowledge and willingness to help of the young people

\section{REFERENCES}

1 European report on preventing violence and knife crime among young people WHO, 2010

2 Recorded Offences Involving the Use of Weapons. Focus on Violent Crime and Sexual Offences, Chapter 3 2012/2013 Release. Office for National Statistics. Feb 2014.

\section{G106(P) INAPPROPRIATE AMBULANCE USE IN PAEDIATRICS}

K Blundell, E Abrahamson. Chelsea and Westminster Hospital, NHS, London, UK

\subsection{6/archdischild-2015-308599.105}

The use of the ambulance service and emergency department for non-urgent needs is considered a significant contributor to health system inefficiencies and a financial stressor. We performed a retrospective audit of children "inappropriately" transferred to a central London paediatric emergency department (PED) by ambulance during the period February to April of 2014. Inappropriate ambulance transfer was defined as children over the age of 2 years who did not require interventions or investigations in the PED, who had not been advised to call an ambulance by 111 or primary care services, and whose presenting complaints or diagnoses did not have any features which might be interpreted as worrying by non-medically trained caregivers. 512 patients were transferred to the PED by ambulance during the study period, of these $102(20 \%)$ were admitted to hospital. $309(60 \%)$ did not require any investigations or interventions (excluding observations and simple analgesia) and of these, 174 (33\%) were thought to represent potentially avoidable and "inappropriate" ambulance transfers. With finite resources and pressures on both the London ambulance service and PED 